\title{
The Onset of NIDDM and its Relationship to Clinical Diagnosis in Egyptian Adults
}

\author{
T.J. Thompsona, M.M. Engelgau", M. Hegazyb, M.A. Alib, E.S. Sous', A. Badran', W.H. Hermanc \\ -The Epidemiology and Statistics Branch, Division of Diabetes \\ Translation, National Center for Chronic Disease Prevention and \\ Health Promotion, Centers for Disease Control and Prevention, \\ Atlanta, USA, the 'Diabetes Institute, Ministry of Health, Cairo, Egypt \\ and 'Department of Internal Medicine, University of Michigan, Ann \\ Arbor, USA
}

\begin{abstract}
The onset of diabetes relative to clinical diagnosis was estimated in Egyptians with noninsulin-dependent diabetes mellitus (NIDDM) based on the relationship between retinopathy and duration of diabetes. Between July 1992 and October 1993 the Diabetes in Egypt (DIE) Project performed a cross-sectional, population-based survey with clinical and laboratory follow-up to describe the prevalence of microvascular, neuropathic, and macrovascular complications among Egyptians $\geqslant 20$ years of age with diagnosed diabetes, previously undiagnosed diabetes, impaired glucose tolerance, and normal glucose tolerance. The sample of persons with diabetes diagnosed prior to the survey had medical examinations which included a dilated eye examination and retinal photographs. Generalized linear models were used to relate the probability of retinopathy to duration of diabetes. Among 218 persons with diabetes diagnosed prior to the DIE project, 87 (40 \%) had diabetic retinopathy. The onset of retinopathy was estimated to occur 2.6 years $(p=0.04)$ prior to clinical diagnosis. The estimated annual incidence of retinopathy was $5 \%$ and the estimated prevalence at the time of clinical diagnosis of diabetes was $12 \%$. On the basis of reports that retinopathy does not occur until approximately 5 years after the development of diabetes, the onset of NIDDM was estimated to occur 7.6 years prior to its clinical diagnosis. This estimate of the onset of NIDDM in Egyptians is comparable to other estimates reported for US and Australian populations.
\end{abstract}

KEY WORDS Non-insulin-dependent diabetes mellitus Diagnosis Retinopathy Epidemiology Egypt

\section{Introduction}

Undiagnosed diabetes is an important clinical and public health problem. World-wide, half of all cases of diabetes are undiagnosed.' The onset of non-insulin-dependent (Type 2) diabetes mellitus (NIDDM) is suspected to precede its clinical diagnosis by several years because diabetic microvascular complications that take years to develop are often present at the time of clinical diagnosis. At clinical diagnosis, as many as $29 \%$ of persons with diabetes have retinopathy, ${ }^{2} 8 \%$ have nephropathy, ${ }^{3}$ and $9 \%$ have neuropathy. ${ }^{4}$

Because the microvascular structures in the eye can be directly examined, retinopathy is usually the first of the diabetic microvascular complications detected. Retinopathy is a sensitive and specific indicator that can be used to estimate the duration of NIDDM and retrospectively determine its onset. Nephropathy and neuropathy are generally defined using arbitrary thresh-

Correspondence to: T.J. Thompson, Centers for Disease Control and Prevention, Mailstop K-10, 4770 Buford Highway NE, Atlanta GA 30341-3724 USA olds, and are less sensitive than retinal abnormalities as markers of diabetic microvascular complications.

This study was designed to examine the relationships among clinical diagnosis, duration of diabetes, and presence of retinopathy. The findings can be used to estimate the actual onset of NIDDM.

Better understanding of the natural history of diabetes may facilitate the development of diabetes control strategies. A recent study ${ }^{2}$ estimated that the onset of retinopathy occurs 4-7 years before clinical diagnosis of NIDDM. Near-normal blood glucose control has recently been shown to delay development and slow progression of the microvascular and neurologic complications of IDDM. ${ }^{5,6}$ It is possible that improved glycaemic control and aggressive cardiovascular risk reduction during the preclinical phase of NIDDM may be more beneficial than later treatment.

\section{Patients and Methods}

The method of identification and description of the study population has been reported elsewhere.? Between July 1992 and October 1993, the Diabetes in Egypt (DIE) Project performed a cross-sectional, population-based 
survey with clinical and laboratory follow-up to describe the prevalence of microvascular, neuropathic, and macrovascular complications among Egyptians $\geq 20$ years of age. Informed consent was obtained from all participants before field workers collected information about diabetes and its duration. Duration of diabetes was defined as time since participants were first told by a physician they had diabetes. All respondents at high risk for diabetes (random capillary glucose $\geq 5.6 \mathrm{mmol}^{-1}$ ) and a random sample of those at low risk for undiagnosed diabetes (random capillary glucose $<5.6 \mathrm{mmol} \mathrm{I}^{-1}$ ) were invited to have a more extensive medical examination done at the Diabetes Institute.

During the DIE Project survey 395 persons with diabetes diagnosed by a physician prior to the DIE project had medical examinations which included an oral glucose tolerance test to confirm diabetes using World Health Organization criteria, ${ }^{8}$ a dilated eye examination by an ophthalmologist, and fundus photographs taken through the dilated pupil of each eye with a Canon retinal camera (CR4-45NM, Canon Inc., Tokyo, Japan).

The fundus photographs were graded by the University of Wisconsin Fundus Photo Reading Centre using a modification of the Airlie House classification scheme for diabetic retinopathy. 9 Retinopathy was considered present when there were retinal microaneurysms either alone or with other nonproliferative changes, when there were preproliferative or proliferative changes, or when vitreous haemorrhage had occurred.

Of the 395 persons with diabetes diagnosed by a physician prior to the DIE Project, 289 had gradable retinal photographs. Those who did not have gradable photographs were similar to those who had gradable retinal photographs. Average age was 53 vs 56 years. Average duration of diabetes was 11 vs 10 years. Insulin use was 19 vs $21 \%$ and the proportion of females was 63 vs $69 \%$. The 10 persons were diagnosed at $<30$ years of age and who used insulin were excluded because they were considered to have IDDM. Fourteen persons had missing data on clinical diagnosis. Additionally, persons with the time since clinical diagnosis $>\mathbf{2 0}$ years $(n=47)$ were excluded because of concerns related to selective mortality. This resulted in a study population of 218 persons.

Logistic regression was used in a preliminary analysis to test if the probability of retinopathy was related to diabetes duration, sex, age, and urban versus rural residence. For the primary analyses we used generalized linear models. ${ }^{10}$ These are a class of statistical models that includes classical linear models (i.e. least squares regression), log-linear models, probit and logit (i.e. logistic regression) models, and several models for survival data. Specification of a model within this class requires a link function and a probability distribution. For example, a log link and Poisson distribution is equivalent to a log-linear model. An identity link and normal distribution is equivalent to least squares regression. A logit link and binomial distribution is equivalent to logistic regression. In this study a complementary log-log link and binomial probability distribution is used. The response variable was the presence of retinopathy. The predictor variable was the logarithm of the quantity: number of years diabetes was present after clinical diagnosis plus the duration of diabetes prior to clinical diagnosis. This model has the form

$$
\ln (-\ln (1-F(t)))=\beta+\alpha \ln \left(t+t_{0}\right)
$$

where $F(t)$ is the probability of retinopathy within time $t$, and $t$ is duration of diabetes since clinical diagnosis. The duration of diabetes prior to clinical diagnosis, $t_{0}$, was estimated using the profile likelihood. ${ }^{10}$ The profile likelihood method involves fitting the model for a series of fixed values of $t_{0}$ and then choosing the model that provides the best fit to the data as measured by the maximized likelihood function. The above model implies that the distribution of time to retinopathy is Weibull. ${ }^{11}$ The Weibull is a flexible survival distribution that includes increasing, decreasing, and constant hazard functions.

The confidence interval $(\mathrm{Cl})$ for duration of diabetes prior to clinical diagnosis was also estimated from the profile likelihood. Confidence intervals for other parameters will be slightly underestimated because $t_{0}$ is treated as known in the computations. This was corrected by using bootstrap methods. ${ }^{12}$ Bootstrap percentile confidence intervals were calculated based on 2000 replications. Confidence intervals of any desired level can be obtained using bootstrap methods.

All the parameters in our final model are known to be greater than zero. Therefore it is natural to think in terms of one-sided p-values and one-sided confidence intervals. However, as a compromise between presenting $95 \%$ one-sided confidence intervals and the more familiar $95 \%$ two-sided confidence intervals, $90 \%$ twosided confidence intervals are presented. Models were fit using the GLIM statistical package ${ }^{11}$ and additional calculations were programmed in GAUSS. ${ }^{13}$

\section{Results}

There were 218 persons with clinically diagnosed NIDDM in the study population. The median age was 54 (range $22-72$ ) years, $33 \%$ were male, $89 \%$ resided in urban areas, and $13 \%$ used insulin. The duration since clinical diagnosis was $1-5$ years for $38 \%, 6-10$ years for $36 \%$, and 11-20 years for $26 \%$. Diabetic retinopathy was present in $\mathbf{4 0} \%$. Duration-specific retinopathy rates were $20 \%$ for $1-5$ years, $51 \%$ for $6-10$ years, and $54 \%$ for 11-20 years.

Based on logistic regression analyses, the probability of retinopathy significantly increased with the duration since clinical diagnosis $(p<0.001)$. Results are presented in Table 1. After adjustment for duration since clinical diagnosis, retinopathy was not related to age $(p=0.6)$, sex $(p=0.8)$, or urban versus rural residence $(p=0.6)$. 
Table 1. Results of fitting a logistic regression model to the retinopathy data $(n=218)$

\begin{tabular}{|c|c|c|c|c|c|}
\hline Variable & $\begin{array}{l}\text { Parameter } \\
\text { estimate }\end{array}$ & $\begin{array}{l}\text { Standard } \\
\text { error }\end{array}$ & $p$-value & Odds ratio & $95 \% \mathrm{Cl}$ \\
\hline Intercept & -0.88 & 1.00 & & & \\
\hline Duration $(\gamma r)$ & 0.12 & 0.03 & $<0.001$ & 1.12 & $(1.06,1.19)$ \\
\hline Age $(y r)$ & -0.01 & 0.02 & 0.6 & 0.99 & $(0.95,1.03)$ \\
\hline Female vs male & 0.09 & 0.31 & 0.8 & 1.09 & $(0.60,2.00)$ \\
\hline Rural vs urban & -0.26 & 0.50 & 0.6 & 0.77 & $(0.29,2.06)$ \\
\hline
\end{tabular}

Our final generalized linear model, plotted in Figure 1, simplified to

$$
F(t)=1-\exp [-0.050(t+2.6)]
$$

The onset of retinopathy was estimated to occur 2.6 years $(90 \% \mathrm{Cl} 0.3-8.4$ years) prior to clinical diagnosis. Testing the hypothesis that the onset of retinopathy began at clinical diagnosis yielded a $p$-value of 0.04 . The estimated annual incidence of retinopathy was $5.0 \%$ (90\% Cl 3.0-7.0\%). The prevalence of retinopathy at diagnosis of diabetes was estimated to be $12 \%(90 \% \mathrm{Cl}$ $1-24 \%)$.

To check the appropriateness of the model, the data were divided into five groups of approximately 40 persons by increasing duration since clinical diagnosis of NIDDM. Prevalence estimates by group versus duration since clinical diagnosis for each observation are included in Figure 1. This shows a reasonable fit to the data. Note the model fitting process used the individual, ungrouped data.

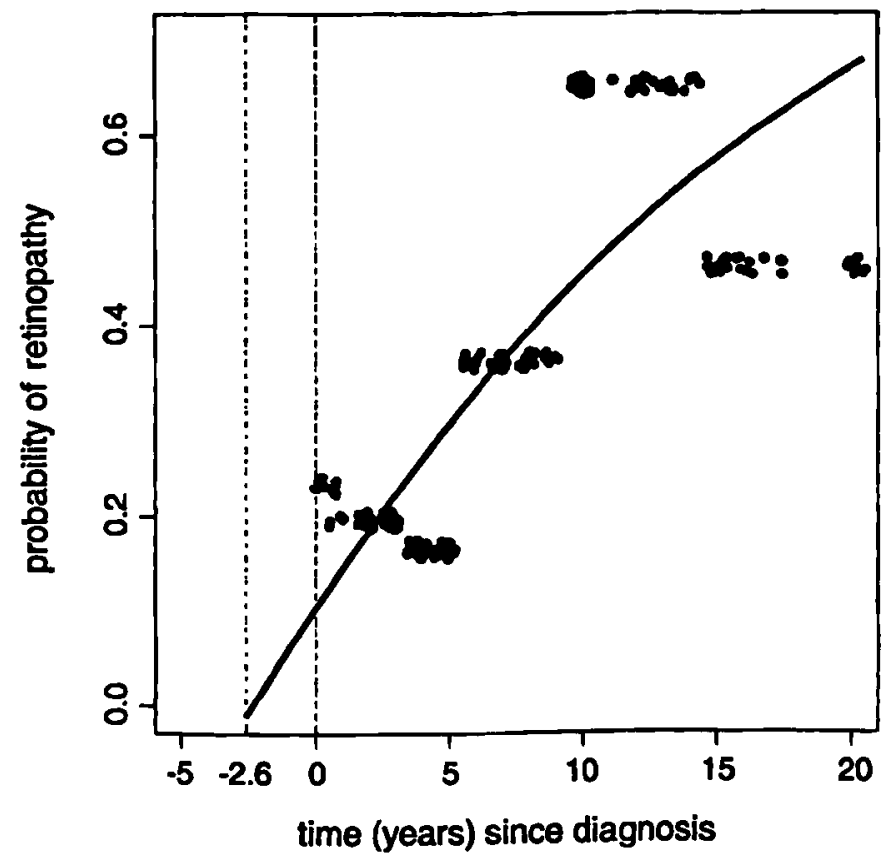

Figure 1. Probability of diabetic retinopathy versus time since diagnosis of diabetes $(n=218)$. The solid line is the model based prediction. The points represent a grouping of the data into five approximately equal size groups

\section{Discussion}

Using the relationship between retinopathy and the time since clinical diagnosis, we estimated that no retinopathy was present in the population 2.6 years prior to the clinical diagnosis of their diabetes. Assuming that diabetic retinopathy does not occur until approximately 5 years after development of diabetes, ${ }^{14}$ we estimated that the onset of diabetes occurs approximately 7.6 years prior to clinical diagnosis. Using these assumptions our estimate of the onset of NIDDM prior to diagnosis is lower than recent reports in US and Australian populations. ${ }^{2}$ However, the confidence interval (0.3-8.4 years) for our estimate includes the values reported for US and Australian populations.

We estimated in this population that at the time of clinical diagnosis the prevalence of diabetic retinopathy was $12 \%$. This estimate of the prevalence of retinopathy is higher than reported in a rural Australian population and lower than that in US' and European populations. ${ }^{15,16}$ Factors that might explain the estimated differences among populations include behaviours related to seeking care, access to medical care, physician practices in pursuing the diagnosis of diabetes, the severity of hyperglycaemia, the prevalence of comorbidities which accelerate complications, and variations in racial and ethnic susceptibility to complications. However, the prevalence of retinopathy in populations where studies include persons detected through active case finding, such as population-based surveys, or intense screening activity, will tend to be lower because diabetes is more likely to be detected at an earlier stage, that is, before the clinical diagnosis is made. Caution must be taken in comparing various studies. In the Egyptian population, the prevalence of retinopathy in persons with diabetes first detected through population-based screening and diagnostic testing during the Diabetes in Egypt survey was $15 \%$.

We also estimate a $47 \% 10$ year incidence rate of retinopathy ( $5 \%$ annual incidence). The WESDR ${ }^{17}$ found a 10-year incidence rate of $79 \%$ in insulin-taking persons 30 years or older and $67 \%$ in non-insulin-taking persons 30 years or older with diabetes. The severity of hyperglycaemia, other comorbidities, and racial ethnic differences may also explain these differences.

The approach we used to predict the onset of NIDDM 
has several practical advantages when compared to other methods. ${ }^{2}$ First, the predicted prevalence of retinopathy is constrained to lie between 0 and $100 \%$. Second, the data do not need to be grouped to fit the model, which is important because the data set analysed here is not large. Third, the duration of diabetes prior to clinical diagnosis is a parameter in the model and is estimated as part of the model fitting process. Furthermore, we excluded persons with duration since clinical diagnosis $>20$ years because there may be higher mortality within this group in persons with more advanced microvascular disease thus introducing bias. Including these persons could lead to an underestimate of annual incidence and an overestimate of duration prior to diagnosis.

Persons with diabetes may benefit from improved glycaemic control and aggressive cardiovascular risk reduction. Thus, more aggressive measures to detect diabetes in earlier stages and initiate comprehensive diabetes management may be warranted. Because risk factors for diabetes, including obesity and sedentary life style are common in Egypt ${ }^{7}$ and increasing elsewhere, ${ }^{18}$ the prevalence of diabetes is expected to increase. The burden of suffering caused by diabetes is large and may increase if interventions are not undertaken.

\section{References}

1. King $H$, Rewers $M$. Global estimates for prevalence of diabetes mellitus and impaired glucose tolerance in adults. Diabetes Care 1993; 16; 157-177.

2. Harris MI, Klein R, Welborn TA, Knuiman MW. Onset of NIDDM occurs at least 4-7 yr before clinical diagnosis. Diabetes Care 1992; 15: 815-819.

3. Ballard DJ, Humphrey LL, Melton LJ, Frohnert PP, Chu PC, O'Fallon WM, et al. Epidemiology of persistent proteinuria in type II diabetes mellitus. Population-based study in Rochester, Minnesota. Diabetes 1988; 37:405-412.

4. Harris MI. Undiagnosed NIDDM: Clinical and public health issues. Diabetes Care 1993; 16: 642-652.
5. The Diabetes Control and Complications Trial Research Group. The effect of intensive treatment of diabetes on the development and progression of long-term complications in insulin-dependent diabetes mellitus. New Eng/ / Med 1993; 329: 977-986.

6. Reichard P, Nilsson BY, Rosenqvist U. The effect of longterm intensified insulin treatment on the development of microvascular complications of diabetes mellitus. New Engl / Med 1993; 329: 304-309.

7. Herman WH, Ali MA, Aubert RE, Engelgau MM, Kenny S], Gunter EW, et al. Diabetes mellitus in Egypt-Risk factors and prevalence. Diabetic Med 1995; 12: 1126-1131.

8. WHO Expert Committee on Diabetes Mellitus. Diabetes Mellitus. Second Report. Technical Report Series 646. Geneva: WHO, 1980: 9-14.

9. Diabetic Retinopathy Study Research Group. VII. A modification of the Airlie House classification of diabetic retinopathy. Invest Ophthalmol Visual Sci 1981; 21: 210-226.

10. McCullagh P, Nelder JA. Generalized Linear Models, 2nd edn. New York: Chapman \& Hall, 1989.

11. Aitkin M, Anderson D, Francis B, Hinde J. Statistical Modelling in GLIM. Oxford: Clarendon Press, 1989.

12. Efron B, Tibshirani RJ. An Introduction to the Bootstrap. New York: Chapman \& Hall, 1993.

13. Aptech Systems Inc. The GAUSS System Version 3.2.5. Maple Valley, WA: Aptech Systems Inc., 1994.

14. Jarrett Jr. Duration of non-insulin-dependent diabetes and development of retinopathy: analysis of possible risk factors. Diabetic Med 1986; 3: 261-263.

15. Aldington SJ, Kohmer EM, Nugent Z. Retinopathy at entry in the United Kingdom Prospective Diabetes Study (UKPDS) of maturity onset diabetes. Diabetic Med 1987; 4: 355.

16. Patrick AW, Leslie PJ, Clark BF, Frier BF. The natural history and associates of microalbuminuria in type 2 diabetes during the first year after diagnosis. Diabetic Med 1990; 7: 902-908.

17. Klein R, Klein BE, Moss SE, Cruickshanks KJ. The Wisconsin epidemiologic study of diabetic retinopathy. XIV. Ten-year incidence and progression of diabetic retinopathy. Arch Ophthal 1994; 112: 1217-1228.

18. Centers for Disease Control and Prevention. Prevalence of selected risk factors for chronic disease by education level in racial/ethnic populations-U.S. Morbidity and Mortality Weekly Report 1994; 43: 894-899. 